\title{
Deformation and shell effects in nuclear mass formulas
}

\author{
César Barbero ${ }^{\mathrm{a}, \mathrm{b}}$, Jorge G. Hirsch ${ }^{\mathrm{c}, *}$, Alejandro E. Mariano ${ }^{\mathrm{a}, \mathrm{b}}$ \\ ${ }^{a}$ Departamento de Física, Universidad Nacional de La Plata, C.C. 67, 1900 La Plata, Argentina \\ b Instituto de Física La Plata, CONICET, 1900 La Plata, Argentina \\ ${ }^{\mathrm{c}}$ Instituto de Ciencias Nucleares, Universidad Nacional Autónoma de México, 04510 México D.F., Mexico
}

Received 31 August 2011; received in revised form 16 November 2011; accepted 17 November 2011

Available online 20 November 2011

\begin{abstract}
We analyze the ability of three different Liquid Drop Mass (LDM) formulas to describe nuclear masses for nuclei in various deformation regions. Separating the 2149 measured nuclear species into eight sets with similar quadrupole deformations, we show that the masses of prolate deformed nuclei are better described than those of spherical ones. In fact, the prolate deformed nuclei are fitted with an RMS smaller than $750 \mathrm{keV}$, while for spherical and semi-magic species the RMS is always larger than $2000 \mathrm{keV}$. These results are found to be independent of pairing.

It is also shown that the macroscopic sector of the Duflo-Zuker (DZ) mass model reproduces shell effects, while most of the deformation dependence is lost and the RMS is larger than in any LDM. Adding to the LDM the microscopically motivated DZ master terms introduces the shell effects, allowing for a significant reduction in the RMS of the fit but still exhibiting a better description of prolate deformed nuclei. The inclusion of shell effects following the Interacting Boson Model's ideas produces similar results. (c) 2011 Elsevier B.V. All rights reserved.
\end{abstract}

Keywords: Nuclear masses; Binding energies; Mass models; Duflo-Zuker

\section{Introduction}

When nuclear physicists refer to the description of nuclear masses employing the Liquid Drop (LD) model formula, we have a contradictory speech. In fact, sometimes we say: "The liquiddrop energy of a spherical nucleus is described by a Bethe-Weizsäcker mass formula" ([1] citing [2]) or "This is a crude model that does not explain all the properties of the nucleus, but

\footnotetext{
* Corresponding author.

E-mail address: hirsch@nucleares.unam.mx (J.G. Hirsch).
} 
does explain the spherical shape of most nuclei" [3]. However, at the same time, we say: "The semi-empirical mass formula gives a good approximation for atomic masses and several other effects, but does not explain the appearance of magic numbers" [3], which are usually considered the 'more spherical' nuclei, because deformation is associated to the quadrupolar interaction between valence protons and neutrons [4]. The usual procedure to find a phenomenological mass formula which reduces the root mean square (RMS) for the actually measured 2149 nuclear species, is to start with the Liquid Drop Mass (LDM) and add to it corrections due to deformation and shell-effects.

The description of nuclear masses in terms of the LDM paved the way to the basic understanding of nuclear properties, like the saturation of the nuclear force, the existence of pairing and shell effects, and the description of fission and fusion processes [5]. The Q-values of different nuclear reactions, obtained from mass differences, must be accurately known to allow the description of the astrophysical origin of the elements [6]. Accurate theoretical predictions of nuclear masses remain a challenge [7], sharing the difficulties with other quantum many-body calculations, and complicated by the absence of a full theory of the nuclear interaction.

Decades of work have produced microscopic and macroscopic mass formulas [8]. At present, the most successful approaches seem to be the microscopic-macroscopic models, like the Finite Range Droplet Model (FRDM) [9], its improvements [10], and the realistic Thomas-Fermi (TF) models $[11,12]$, the Skyrme and Gogny Hartee Fock Bogolyubov (HFB) $[13,14]$, and the Duflo-Zuker (DZ) mass formula [15-17]. They allow for the calculation of masses, charge radii, deformations, and in some cases also fission barriers. They all contain a macroscopic sector which resembles the LDM formula, and include deformation effects. HFB calculations are now able to fit known nuclear masses with deviations competitive with the microscopic-macroscopic calculations, while the most precise and robust nuclear mass predictions are given by the DZ model [8,18], which gives an RMS of $373 \mathrm{keV}$.

Efforts for building algebraic nuclear mass formulas inspired by the DZ model's success have led to detailed analysis of the microscopic building blocks of this model [19], which suggested new ways to introduce the shell effects through the DZ master terms [20]. Other line of thought connected the Interacting Boson Model (IBM) F-spin with the DZ microscopic terms [21,18,22], which allows for very good fits of the nuclear binding energies when an additional one-body Hamiltonian with a large number of parameters is employed [23].

Following these works, we perform in this paper an analysis of the interplay between deformations and shell effects for different nuclear mass formulas. We start studying the ability of LDM to describe nuclear masses for nuclei in different deformation regions and explicitly show that the best fit is obtained for deformed nuclei, extending a previous preliminary work [24]. These results are found to be independent of the pairing term, which could be failing in the vicinity of closed shell nuclei.

Analyzing the macroscopic terms of the DZ mass model we show that shell effects are reproduced and most of the deformation dependence is lost while the RMS is larger than in any LDM. Adding to the LDM the microscopically motivated DZ master terms introduces the shell effects, allowing for a significant reduction in the RMS of the fit but still exhibiting a better description of prolate deformed nuclei. The inclusion of shell effects through a dependence on the number of valence nucleons produces similar results.

The paper is organized as follows: the fits for three LDM are presented in Section 2, and the DZ and other microscopic algebraic estimations are discussed in Section 3. A meticulous analysis of the master terms is performed in Section 4, and a comparison with other estimations based on IBM is given in Section 5. Conclusions are drawn in Section 6. 


\section{The fits for three LDM formulas}

We have selected three LDM formulas to analyze their ability to fit nuclear masses.

\section{1. $L D M 1$}

The first one is an improved version of the LDM formula with modified symmetry and Coulomb terms, built following a consistent treatment of nuclear bulk and surface effects [22]. The negative nuclear binding energy is given by

$$
E_{\mathrm{LDM} 1}=-a_{v} A+a_{s} A^{2 / 3}+S_{v} \frac{4 T(T+1)}{A\left(1+y A^{-1 / 3}\right)}+a_{c} \frac{Z(Z-1)}{(1-\Lambda) A^{1 / 3}}-a_{p} \frac{\Delta}{A^{1 / 3}},
$$

where: (i) the pairing interaction is given by $\Delta=2,1$, and 0 for even-even, odd-mass and odd-odd nuclei, respectively; (ii) a correction to the radius of the nucleus is included through a modification $\Lambda$ in the Coulomb term, $\Lambda=\frac{N-Z}{6 Z\left(1+y^{-1} A^{1 / 3}\right)}$; (iii) the symmetry term employs $4 T(T+1)$, with $T=|N-Z| / 2$, instead of $(N-Z)^{2}$ to account for the Wigner energy; and (iv) the Coulomb interaction is proportional to $Z(Z-1)$ to avoid the Coulomb interaction of a proton with itself.

\section{2. $L D M 2$}

The second LDM formula is a modified version of the Bethe-Weizsäcker one, which incorporates explicitly isospin effects [1]:

$$
E_{\mathrm{LDM} 2}=-a_{v} A+a_{s} A^{2 / 3}+a_{s y m} I^{2} A+a_{c} \frac{Z(Z-1)}{A^{1 / 3}}\left(1-Z^{-2 / 3}\right)-a_{\text {pair }} \frac{\delta_{n p}}{A^{1 / 3}},
$$

with isospin asymmetry $I=(N-Z) / A$. The pairing term is taken from [19]

$$
\delta_{n p}= \begin{cases}2-|I|: & N \text { and } Z \text { even, } \\ |I|: & N \text { and } Z \text { odd, } \\ 1-|I|: & N \text { even, } Z \text { odd, and } N>Z, \\ 1-|I|: & N \text { odd, } Z \text { even, and } N<Z, \\ 1: & N \text { even, } Z \text { odd, and } N<Z, \\ 1: & N \text { odd, } Z \text { even, and } N>Z,\end{cases}
$$

and the symmetry energy coefficient, including an $I$ correction on the conventional surfacesymmetry term of LDM to approximately describe the Wigner effect for heavy nuclei, is written as

$$
a_{\text {sym }}=c_{\text {sym }}\left[1-\frac{\kappa}{A^{1 / 3}}+\frac{2-|I|}{2+|I| A}\right] .
$$

The Coulomb exchange and surface diffuseness corrections to the Coulomb energy are approximately taken into account through the term $Z^{-2 / 3}[1]$.

\section{3. $L D M 3$}

The third LDM formula is derived from the liquid drop model with the pairing energies of the Thomas-Fermi model [25] 
Table 1

The nine groups of nuclei employed in the present study, their range of quadrupole deformation, and their number of nuclei.

\begin{tabular}{llllllllll}
\hline Group & All & 1 & 2 & 3 & 4 & 5 & 6 & 7 & Semi-magic \\
\hline$e_{2} \min$ & -0.65 & -0.65 & -0.11 & 0.00 & 0.04 & 0.12 & 0.18 & 0.23 & \\
$e_{2} \max$ & 0.65 & -0.11 & 0.00 & 0.04 & 0.12 & 0.18 & 0.23 & 0.65 & \\
$N_{\text {nucl }}$ & 2149 & 258 & 252 & 332 & 272 & 307 & 364 & 364 & 185 \\
\hline
\end{tabular}

$$
\begin{aligned}
E_{\mathrm{LDM} 3}(N, Z)= & -a_{v}\left(1-k_{v} I^{2}\right) A+a_{s}\left(1-k_{s} I^{2}\right) A^{2 / 3}+a_{k}\left(1-k_{k} I^{2}\right) A^{1 / 3}+\frac{3}{5} \frac{e^{2} Z^{2}}{r_{0} A^{1 / 3}} \\
& -f_{p} Z^{2} / A-a_{c, e x c} Z^{4 / 3} / A^{1 / 3}+E_{\text {pair }} .
\end{aligned}
$$

It includes: (i) a first term representing the volume energy corresponding to the saturated exchange force and infinite nuclear matter, with $I^{2} A$ being the asymmetry energy of the BetheWeizsäcker mass formula; (ii) a surface energy corresponding to semi-infinite nuclear matter and originated by the deficit of binding energy of the nucleons at the nuclear surface; (iii) a curvature energy resulting from non-uniform properties which correct the surface energy and depends on the mean local curvature; (iv) the decrease of binding energy due to the Coulomb repulsion; (v) a $Z^{2} / A$ diffuseness correction to the sharp radius Coulomb energy; (vi) a $Z^{4 / 3} / A^{1 / 3}$ charge exchange correction term; (vii) the pairing energies $E_{\text {pair }}$ of the Thomas-Fermi model (taken from Eq. (A.2) in Ref. [11]).

\subsection{The fits}

The coefficients of the three LD models were selected to minimize the RMS when the predicted binding energies $B E_{\text {th }}(N, Z)$ are compared with the experimental ones $B E_{\exp }(N, Z)$, reported in AME03 [26], modified so as to include more realistically the electron binding energies as explained in Appendix A of Lunney, Pearson and Thibault [8]:

$$
\mathrm{RMS}=\left\{\frac{\sum\left[B E_{\exp }(N, Z)-B E_{\mathrm{th}}(N, Z)\right]^{2}}{N_{\text {nucl }}}\right\}^{1 / 2} .
$$

The minimization procedure uses the routine Minuit [27].

The fits were performed separating the nuclei in nine groups:

- the first one contains all nuclei whose measured masses are reported in AME03 [26], which have $N, Z \geqslant 8$,

- the next seven groups contain the nuclei whose quadrupole deformations $e_{2}$, taken form the FRDM [9], lie in the ranges listed in the second and third row of Table 1,

- the last group contains all semi-magic nuclei, having $Z=14,28,50,82$ or $N=14,28,50$, 82 or 126.

$N_{\text {nucl }}$ is the number of nuclei in each group, listed in the fourth row of Table 1 . We remark that regions 1 to 7 contain approximately the same quantity of nuclei. Notice that group 1 contains most of the oblate nuclei, that the more spherical nuclei belong to groups 2, 3 and 4 , and that the more prolate deformed nuclei are included in groups 6 and 7. We show in Fig. 1 the different regions in the $N-Z$ plane. Semimagic nuclei are displayed along straight thick black lines. Around 


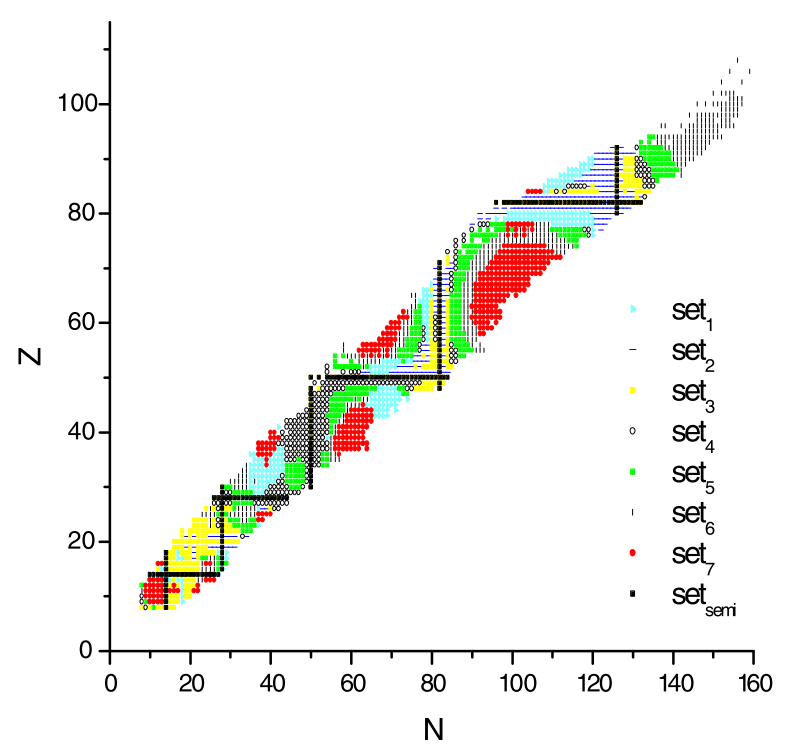

Fig. 1. The seven deformation regions and the semimagic nuclei, shown in the $N-Z$ plane.

Table 2

RMS (in keV) of the individual fits for each of the nine groups, employing Eqs. (1), (2) and (5), respectively, and employing the parameters from the global fit in each region (second line, in parenthesis).

\begin{tabular}{llllllllll}
\hline & All & 1 & 2 & 3 & 4 & 5 & 6 & 7 & Semi-magic \\
\hline LDM1 & 2387 & 1313 & 1676 & 2063 & 1746 & 1053 & 870 & 746 & 2113 \\
& & $(1842)$ & $(3814)$ & $(3080)$ & $(2037)$ & $(1495)$ & $(1815)$ & $(2060)$ & $(3888)$ \\
LDM2 & 2374 & 1254 & 1675 & 2069 & 1762 & 1021 & 838 & 656 & 2056 \\
& & $(1788)$ & $(3733)$ & $(3081)$ & $(2044)$ & $(1502)$ & $(1855)$ & $(2058)$ & $(3916)$ \\
LDM3 & 2422 & 1183 & 1597 & 2151 & 1517 & 986 & 819 & 629 & 1967 \\
& & $(1884)$ & $(3773)$ & $(3186)$ & $(2031)$ & $(1519)$ & $(1861)$ & $(2125)$ & $(3937)$ \\
\hline
\end{tabular}

them cluster the nuclei classified as the more spherical, while the more prolate-deformed ones form closed regions with many valence protons and neutrons.

For each LDM equation, nine fits were performed, one for each group of nuclei. In this way, nine sets of parameters were obtained, which minimize the RMS of each group of nuclei. The values of these parameters can be found in Tables 7, 8 and 9 of Appendix A (see also Ref. [24]). We present in Table 2 the RMS obtained for all groups employing these nine sets of parameters. For completeness, we show also in a second line, between parenthesis, the RMS obtained for each region using the parameters of the global fit ( set $_{\text {all }}$ ).

Table 2 results show that for the three LDM models the masses of prolate deformed nuclei can be described with remarkable precision, with an RMS smaller than $750 \mathrm{keV}$, while the masses of spherical and semi-magic nuclei are those worst described, with RMS larger than $2000 \mathrm{keV}$. It completely challenged the authors preconception that the LDM was best suited to describe spherical nuclei, and that deformation effects were a crucial element necessary to improve the LDM description of nuclear binding energies. As can be read from Table 2, deformed regions are 
Table 3

Number of nuclei with even $N$-even $Z\left(N_{\text {nucl }}^{e e}\right)$, even $N$-odd $Z\left(N_{\text {nucl }}^{e o}\right)$, odd $N$-even $Z\left(N_{\text {nucl }}^{o e}\right)$ and odd $N$-odd $Z$ $\left(N_{\text {nucl }}^{\text {oo }}\right)$.

\begin{tabular}{llllllllll}
\hline Group & All & 1 & 2 & 3 & 4 & 5 & 6 & 7 & Semi-magic \\
\hline$N_{\text {nucl }}^{e e}$ & 576 & 62 & 35 & 154 & 53 & 80 & 104 & 88 & 95 \\
$N_{\text {nucl }}^{e o}$ & 534 & 67 & 74 & 67 & 79 & 73 & 89 & 85 & 40 \\
$N_{\text {nucl }}^{\text {ne }}$ & 536 & 69 & 60 & 80 & 62 & 78 & 89 & 98 & 50 \\
$N_{\text {nucl }}^{\text {no }}$ & 503 & 60 & 83 & 31 & 78 & 76 & 82 & 93 & 0 \\
\hline
\end{tabular}

Table 4

RMS (in keV) of the individual fits for each of the nine groups (columns) in the ee, eo, oe and oo regions, employing Eq. (1) without pairing, and employing the parameters from the global fit in each region (second line, in parenthesis).

\begin{tabular}{llllllllll}
\hline & All & 1 & 2 & 3 & 4 & 5 & 6 & 7 & Semi-magic \\
\hline Even $N$-even $Z$ & 2418 & 1158 & 1443 & 2272 & 1529 & 1050 & 805 & 786 & 2194 \\
& & $(1931)$ & $(3952)$ & $(3158)$ & $(1627)$ & $(1423)$ & $(1792)$ & $(2180)$ & $(3833)$ \\
Even $N$-odd $Z$ & 2404 & 1387 & 1834 & 1698 & 1726 & 1036 & 867 & 712 & 1483 \\
& & $(1913)$ & $(3819)$ & $(2893)$ & $(1964)$ & $(1623)$ & $(1908)$ & $(2121)$ & $(4225)$ \\
Odd $N$-even $Z$ & 2360 & 1382 & 1514 & 1936 & 1638 & 990 & 880 & 749 & 1871 \\
& & $(1914)$ & $(3924)$ & $(2949)$ & $(2000)$ & $(1482)$ & $(1780)$ & $(2051)$ & $(3690)$ \\
Odd $N$-odd $Z$ & 2404 & 1430 & 1370 & 1595 & 1888 & 1072 & 913 & 703 & - \\
& & $(1909)$ & $(3682)$ & $(3307)$ & $(2292)$ & $(1485)$ & $(1933)$ & $(1917)$ & $(-)$ \\
\hline
\end{tabular}

very well adjusted by the three LDM formulas, and the regions around shell closures are those which represent a challenge.

The second line for each LDM formula in Table 2 offers also valuable information. Employing the parameters obtained from the global fit, the largest RMS, close to $4 \mathrm{MeV}$, are found for the semimagic nuclei and those nearly spherical in region 2, which together with those in region 3 have in all cases an RMS larger than $3 \mathrm{MeV}$, confirming that the masses of spherical nuclei are those worst fitted by any LDM. On the other hand, the best fits are found in the moderately prolate nuclei belonging to region 5, with values around 1.5 MeV. All masses in deformed regions are fitted with an RMS smaller than 2.2 MeV.

Given that the description of pairing effects for nuclei with few valence nucleons would require more than a simple parametrization as the one employed here, we have removed odd-even effects from our analysis, separating the nuclei in four additional sets, corresponding to even $N$-even $Z$ (ee), even $N$-odd $Z$ (eo), odd $N$-even $Z$ (oe) and odd $N$-odd $Z$ (oo). The number of nuclear species of each type in each set is indicated in Table 3. We repeated the fits for each ee, eo, oe and oo set in the nine regions, employing the LDM1 mass formula but without the pairing term (last term in Eq. (1)). We arrive to the RMS indicated in Table 4, which one more time indicate that prolate deformed nuclei in region 7 are far better described than spherical ones, regions 2, 3, 4 and semimagic. It shows that the difficulty of the LDM mass formulas to describe spherical nuclei is not at all associated with any odd-even effect, or with the way the pairing contribution is parameterized. In the second line of each set the RMS obtained using the parameters obtained from the global fit are displayed. The removal of pairing effects does not reflects in any noticeable change in their behavior.

In what follows we explore the ability of the algebraic extensions of the LDM to include shell effects in the description of nuclear masses. 


\section{The Duflo-Zuker inspired mass models}

In this section two different mass models, both based on Duflo-Zuker ideas, are discussed in detail. The master terms are introduced in two alternative ways in order to describe the shell effects in mass models, and their correlations with the nuclear deformation is analyzed.

\subsection{DZ1}

The macroscopic sector of the simplest version of the DZ mass model contains six terms leading asymptotically to a LD form [19] (see Section 3.3 below)

$$
E_{\mathrm{DZ} 1}=a_{1}\left(M^{H O}+S\right)-a_{2} \frac{M^{H O}}{\rho}-a_{3} V_{C}-a_{4} V_{T}+a_{5} V_{T S}+a_{6} V_{P} .
$$

The master $M^{H O}$ term is

$$
\begin{aligned}
M^{H O} & =\frac{1}{2 \rho}\left[\left(\sum_{p} \frac{n_{v}+n_{\pi}}{\sqrt{D_{p}^{H O}}}\right)^{2}+\left(\sum_{p} \frac{n_{v}-n_{\pi}}{\sqrt{D_{p}^{H O}}}\right)^{2}\right] \\
& =\frac{1}{\rho}\left[\left(\sum_{p} \frac{n_{v}}{\sqrt{D_{p}^{H O}}}\right)^{2}+\left(\sum_{p} \frac{n_{\pi}}{\sqrt{D_{p}^{H O}}}\right)^{2}\right],
\end{aligned}
$$

where the sums run over all occupied proton and neutron orbitals up to the Fermi level, $D_{p}^{H O}=(p+1)(p+2)$ is the degeneracy of the major harmonic-oscillator (HO) shell of principal quantum number $p$, and $n_{v}(p), n_{\pi}(p)$ are the number of neutrons and protons, respectively, in the HO shell $p$.

It is relevant to mention that this form describes the dominant contribution of the monopole part of the nuclear Hamiltonian. In order to change the HO closures (at $N, Z=8,20,40,70, \ldots$ ) into the observed extruder-intruder (EI) ones at $N, Z=14,28,50,82$ and 126, the $S$ operator proposed by Duflo, given in Eq. (18) of Ref. [19], is employed.

The scaling factor is

$$
\rho=A^{1 / 3}\left[1-\left(\frac{T}{A}\right)^{2}\right]^{2}
$$

The Coulomb, asymmetry and surface asymmetry terms are [19], respectively

$$
\begin{aligned}
& V_{C}=\frac{-Z(Z-1)+0.76\left[Z(Z-1)^{2 / 3}\right]}{r_{c}}, \quad r_{c}=A^{1 / 3}\left[1-\left(\frac{T}{A}\right)^{2}\right] \\
& V_{T}=\frac{4 T(T+1)}{A^{2 / 3} \rho}, \\
& V_{T S}=\frac{4 T(T+1)}{A^{2 / 3} \rho^{2}}-\frac{4 T\left(T-\frac{1}{2}\right)}{A \rho^{4}} .
\end{aligned}
$$

The pairing term $V_{P}$ is the same employed in LDM2. 


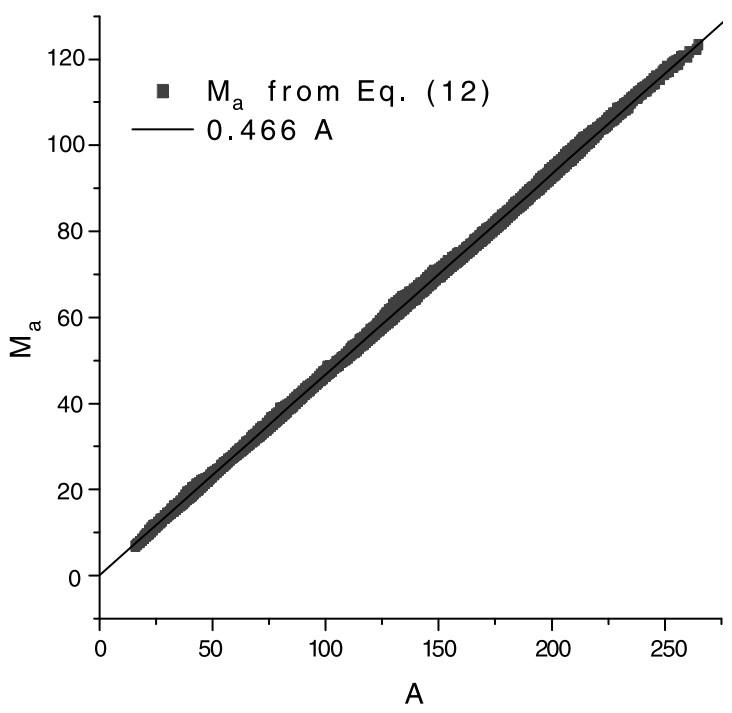

Fig. 2. Master term from DZ model as function of $A$ : gray squares indicate the results calculated with Eq. (12) and solid black line is a linear fit of them.

\section{2. $D Z 2$}

As there exists some uncertainty in the parameterization of the monopole part of the nuclear Hamiltonian [19], in Ref. [20] a modified master term $M$ was proposed, which directly includes the EI shell closures. It is built using an expression similar to (8), but with the index $p$ now referring to the EI major shell with degeneracies $D_{p} \rightarrow D_{p}^{H O}+2$.

$$
E_{\mathrm{DZ} 2}=a_{1} M_{a}-a_{2} \frac{M_{a}}{\rho}-a_{3} V_{C}-a_{4} V_{T}+a_{5} V_{T S}+a_{6} V_{P}
$$

with the new master term:

$$
M_{a}=\frac{1}{\rho}\left(e_{1 v}^{2}+e_{1 \pi}^{2}\right), \quad e_{1 v}=\sum_{p_{v}} \frac{n_{v}}{\sqrt{D_{p_{v}}}}, e_{1 \pi}=\sum_{p_{\pi}} \frac{n_{\pi}}{\sqrt{D_{p_{\pi}}}},
$$

where $D_{p_{v, \pi}}=\left(p_{v, \pi}+1\right)\left(p_{v, \pi}+2\right)+2$ contains the $H O \rightarrow E I$ information and $n_{v}\left(p_{\nu}\right), n_{\pi}\left(p_{\pi}\right)$ are the number of neutrons and protons in their respective EI shell.

\subsection{The master term}

The master term $M_{a}$ contains the microscopic information about shell closures. We show in Fig. 2 its behavior as a function of $A$, evaluated for all the nuclei with $N, Z \geqslant 8$ with measured masses reported in AME03. It scales as a linear function in $A$. Therefore, we can interpret this term as a volume one and, consequently, the term $M_{a} / \rho$ can be associated to a surface one. This allows us to consider the macroscopic sector of the DZ mass formula as an extension of the LDM.

The building blocks of the master term $M_{a}$ in Eq. (12) are $e_{1 v}$ and $e_{1 \pi}$. In Fig. 3 the behavior of $e_{1 v}$ as a function of $N$ is presented, evaluated for all the nuclei with $N, Z \geqslant 8$ with measured masses reported in AME03. The thin black line represents the asymptotic form 


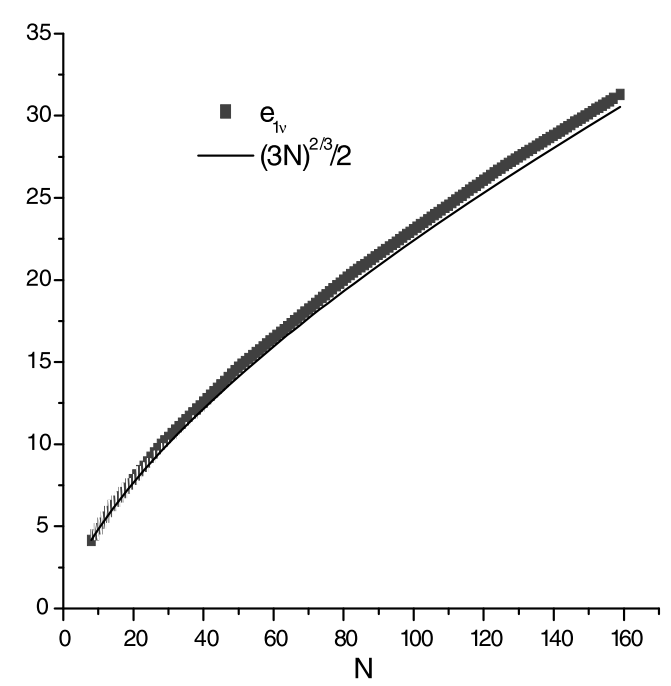

Fig. 3. Dependence of $e_{1 v}$ with $N$.

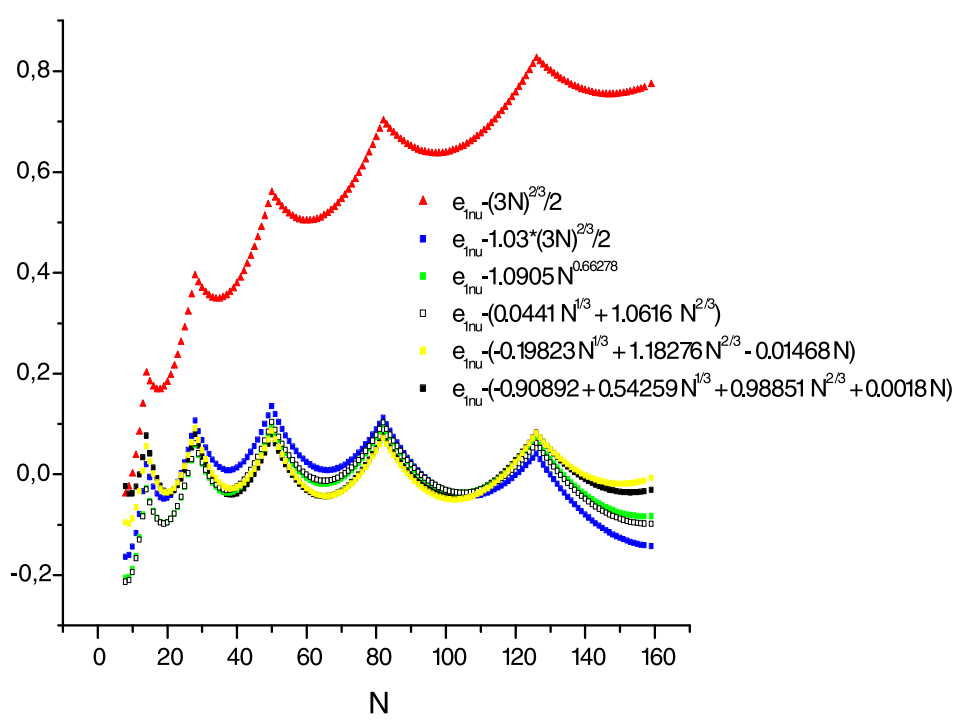

Fig. 4. Difference between $e_{1 v}$ and different approximations to describe its asymptotic behavior as a function of $N$.

$e_{1 v} \approx \frac{(3 N)^{2 / 3}}{2}[20]$. The plot confirms that $e_{1 v}$ scales as $N^{2 / 3}$, with minor differences for small $N$. To visualize this deviation, the difference between $e_{1 v}$ and this asymptotic behavior is presented in Fig. 4 (black triangles). The microscopic elements introduced through $e_{1 v}$ are made evident in this plot. When the asymptotic behavior is removed, shell effects emerge, with well-defined peaks at shell closures. However, there is a remnant continuous increase with $N$ which has not a linear dependence. The description of the asymptotic form of $e_{1 v}$ can be improved by including terms with different powers of $N^{1 / 3}$. The differences between $e_{1 v}$ and these asymptotic forms are displayed with different symbols in Fig. 4. From these results we conclude that a better de- 


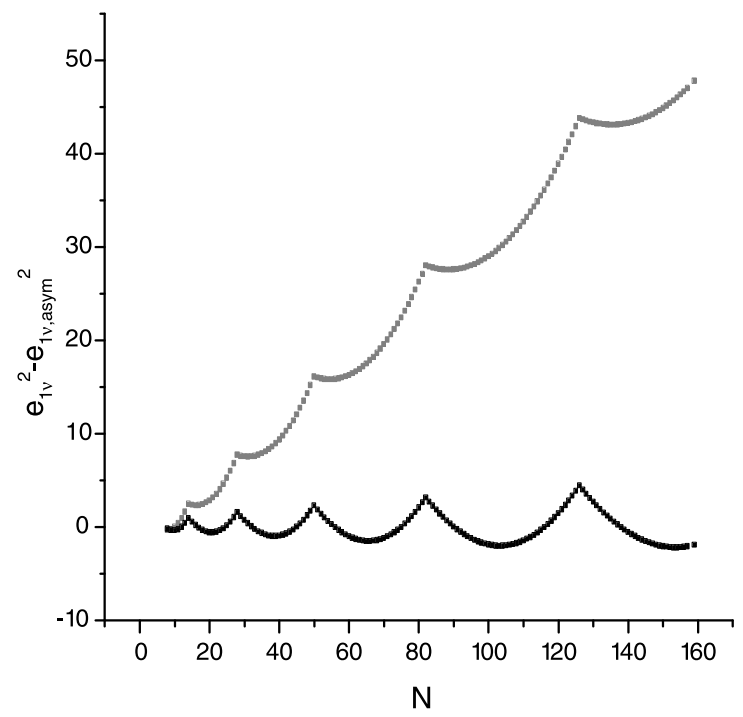

Fig. 5. Difference between $e_{1 v}^{2}$ and its asymptotic behavior for the approximation $e_{1 v} \approx \frac{(3 N)^{2 / 3}}{2}$ (gray line) and for Eq. (13) (black line).

scription can be reached if we adopt the new asymptotic form

$$
e_{1 v, \text { asym }}=-0.90892+0.54259 N^{1 / 3}+0.98851 N^{2 / 3}+0.0018 N .
$$

A similar analysis was performed around Fig. 3 of Ref. [19].

In the construction of the master term $M_{a}$, Eq. (12), we employ $e_{1 v}^{2}$. It is compared with its asymptotic form $e_{1 v \text {, asym }}^{2}$ in Fig. 5. The advantages of using Eq. (13) instead of the simpler $\frac{(3 N)^{2 / 3}}{2}$ expression can be clearly observed: we obtain a global leveling besides the good description of closed shell effects.

Thus the master term $M_{a}$ has the asymptotic behavior described by

$$
M_{a, \text { asym }}=\frac{1}{\rho}\left(e_{1 v, \text { asym }}^{2}+e_{1 \pi, \text { asym }}^{2}\right) .
$$

In what follows we report the results obtained employing the asymptotic form with four terms, which provides the best fit. However, in future work it could make sense to use the simpler one or two term expressions, which reduce the number of parameters introduced in the formalism while still providing a reasonable good asymptotic description, as can be seen in Fig. 4.

\subsection{The fits}

Employing the two versions of the macroscopic sector of the DZ mass model, Eqs. (7) and (11), we have fitted the nuclear masses in the nine regions described above.

The fitting procedure in the different deformation regions leads to the RMS displayed in Table 5. For both models the RMS obtained in each region employing the parameters of the global fit are presented in a second line, between parenthesis. These results show that the ability of both models to describe masses of nuclei in spherical, prolate and semi-magic groups are now comparable. The global RMS are larger than those obtained with the LDM formulas. It is hard to find 
Table 5

RMS (in keV) of the individual fits for each of the nine groups, using Eqs. (7) and (11), and employing the parameters from the global fit in each region (second line, in parenthesis).

\begin{tabular}{llllllllll}
\hline & All & 1 & 2 & 3 & 4 & 5 & 6 & 7 & Semi-magic \\
\hline DZ1 & 2852 & 994 & 1969 & 1557 & 1392 & 2237 & 2562 & 1529 & 1392 \\
& & $(1475)$ & $(2670)$ & $(3026)$ & $(2466)$ & $(2887)$ & $(3287)$ & $(3293)$ & $(2616)$ \\
DZ2 & \multirow{2}{*}{3443} & 1425 & 1544 & 2167 & 1717 & 1729 & 2047 & 2107 & 1973 \\
& & $(1516)$ & $(3431)$ & $(4007)$ & $(2924)$ & $(2295)$ & $(3562)$ & $(4696)$ & $(4160)$ \\
\hline
\end{tabular}
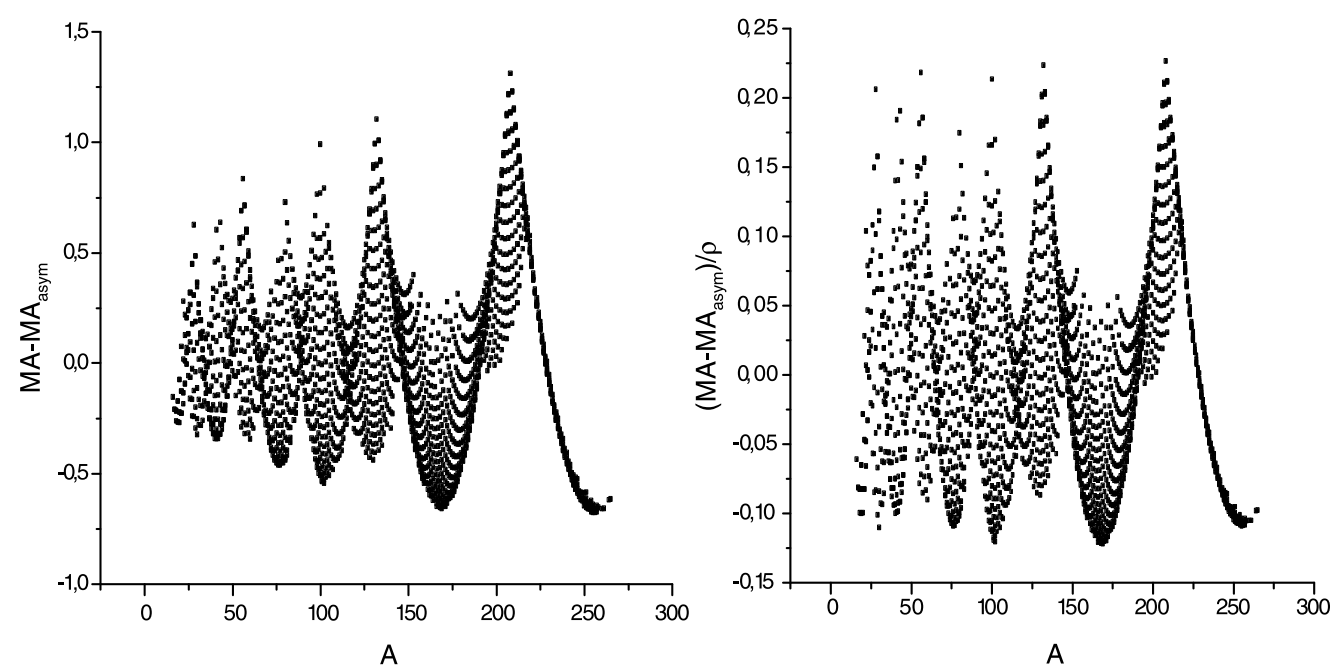

Fig. 6. Volume $\left(M_{a}-M_{a, \text { asym }}\right)$ and surface $\left(\left(M_{a}-M_{a, \text { asym }}\right) / \rho\right)$ terms as function of $A$.

any correlation between the RMS and the regions with different deformations. This fact is also reflected in the values of the fitted parameters, presented in Tables 10 and 11 of Appendix A, because some of them (mainly $a_{5}$ and $a_{6}$ ) vary noticeably from one region to the other.

\section{Adding shell corrections to the LDM}

\subsection{LDM1 plus DZ}

It is possible to combine in a single mass formula both the ability of the LDM to describe the masses of deformed nuclei, and of DZ to include shell effects. To this end, we will work with a LDM, adding the shell effects present in the DZ master term, but with their asymptotic behavior removed, because it is basically contained in the volume and surface terms of the LDM. We have selected as the starting point the LDM1, which has a global RMS smaller than those of DZ1 and DZ2.

Based on our previous analysis, the shell effects will be introduced by a "volume" and "surface" shell corrections defined as $M_{a}-M_{a, \text { asym }}$ and $\left(M_{a}-M_{a, a s y m}\right) / \rho$, respectively. They are plotted in Fig. 6 as a function of $A$, for all nuclei with measured masses reported in AME03.

They resemble the shell effects not included in the LDM. To help the visualization of the shell effects introduced by these differences, we show in Fig. 7 the same terms as function of $N, Z$ 

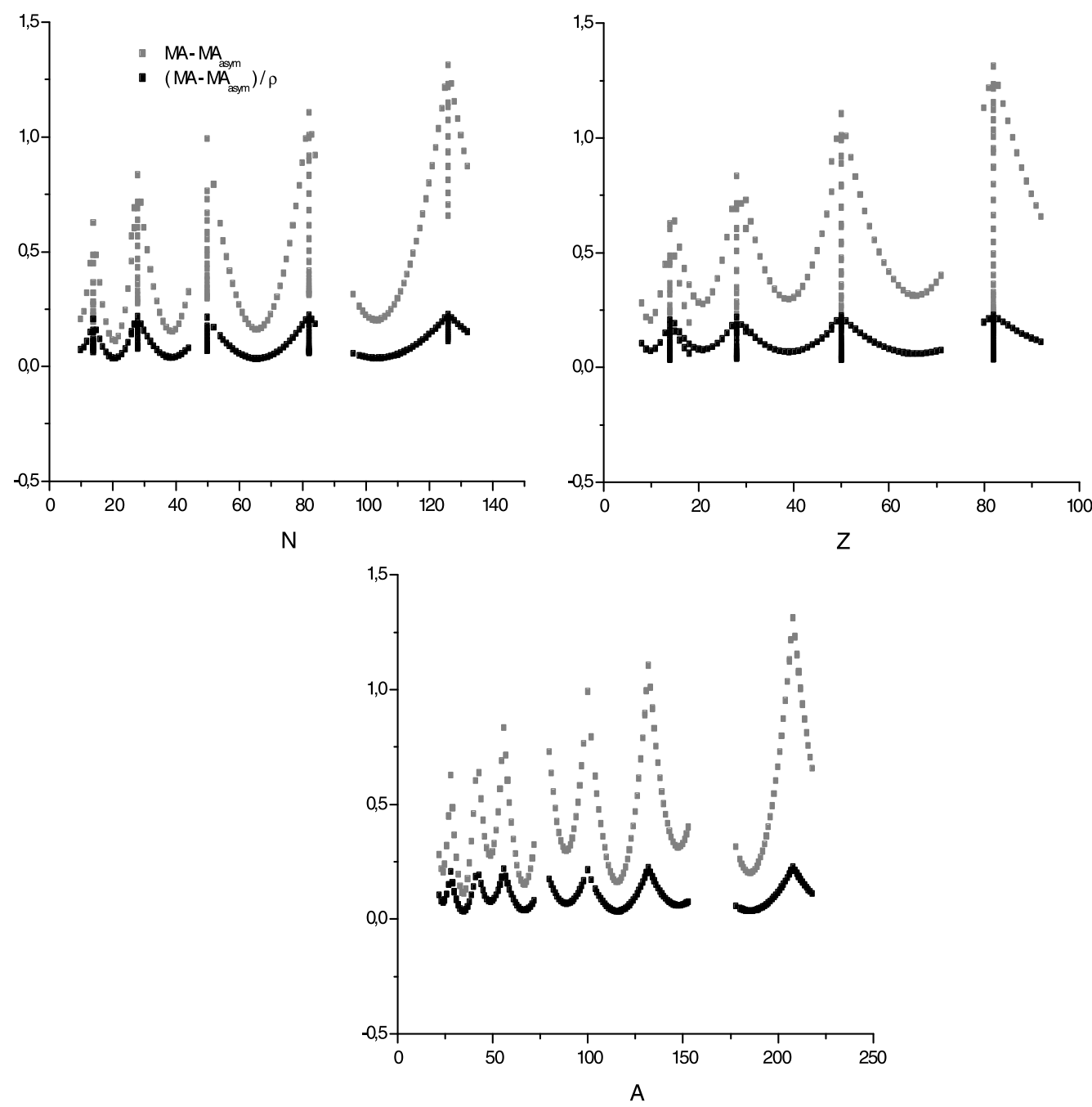

Fig. 7. Volume $\left(M_{a}-M_{a, \text { asym }}\right)$ and surface $\left(\left(M_{a}-M_{a, \text { asym }}\right) / \rho\right)$ terms for semi-magic nuclei.

and $A$ only for semi-magic nuclei, where we can observe one more time the clear peaks at the magic numbers.

As explained above, we have added the shell effects to the LDM1 to construct a new mass formula:

$$
E_{\mathrm{LDM} 1+\mathrm{DZ}}=\mathrm{LDM} 1+a_{\mathrm{vol}}\left(M_{a}-M_{a, a s y m}\right)+a_{\text {surf }}\left(M_{a}-M_{a, a s y m}\right) / \rho .
$$

\subsection{Comparison with other estimations of shell effects}

Inspired by the F-spin symmetry of the IBM's [21,22], shell effects have been recently introduced in extensions of the LDM by adding two terms, linear and quadratic in the number of valence protons, $z_{v}$, and neutrons, $n_{v}[21,18,22]$. The proposed expression for the nuclear 
Table 6

RMS (in keV) of the individual fits for each of the nine groups, using Eqs. (15) and (16), and employing the parameters from the global fit in each region (second line, in parenthesis).

\begin{tabular}{llllllllll}
\hline & All & 1 & 2 & 3 & 4 & 5 & 6 & 7 & Semi-magic \\
\hline LDM1 + DZ & 1407 & 668 & 907 & 1026 & 755 & 784 & 791 & 647 & 952 \\
& & $(1139)$ & $(1529)$ & $(1626)$ & $(1361)$ & $(1441)$ & $(1422)$ & $(1270)$ & $(1703)$ \\
LDM1 + val & \multirow{2}{*}{1075} & 796 & 981 & 1006 & 828 & 711 & 836 & 615 & 1037 \\
& & $(953)$ & $(1230)$ & $(1273)$ & $(1039)$ & $(963)$ & $(1077)$ & $(954)$ & $(1212)$ \\
\hline
\end{tabular}

binding energies is

$$
E_{\mathrm{LDM} 1+\mathrm{val}}=\mathrm{LDM} 1+b_{1}\left(n_{v}+z_{v}\right)+b_{2}\left(n_{v}+z_{v}\right)^{2} .
$$

As the valence occupation numbers are defined as the absolute value of the difference between the actual number of protons or neutrons and the nearest closed shell magic number, the shell closures are implicitly introduced as new parameters at this stage.

\subsection{The fits}

Repeating the analysis for the same nine groups, we obtain the results shown in Table 6 for the RMS employing the two models.

As expected, the improvements in the inclusion of shell effects reduce the global RMS from $2387 \mathrm{keV}$ to 1407 (1075) $\mathrm{keV}$ when the LDM1 + DZ (LDM1 + val) model is used. The results still show a visible tendency to describe better the deformed than spherical nuclei, both for the individual fits and employing the parameters of the global fit in each region. Besides, the results obtained with both formulas look very similar, with a smaller global RMS in the valence model and some advantage of the LDM1 + DZ model to describe semi-magic nuclei.

The parameters set obtained in both fits are presented in Appendix A, in Tables 12 and 13. The relative stability of the parameters $b_{1}$ and $b_{2}$ in the LDM1 + val model, shown in the last two columns of Table 13, is behind the comparatively small global RMS. On the other hand, the shell surface and volume coefficients $a_{\text {surf }}$ and $a_{\mathrm{vol}}$ of the LDM1 + DZ model listed in the last two columns of Table 12 vary both in magnitude and in sign from one deformation region to another. Being a limitation for a good global fit, it offers at the same time the opportunity to relate these parameters with the deformation, a challenge which is left for future work.

Finally, to complete the comparison, we exhibit in Fig. 8 the difference between experimental data $\left(B_{\exp }\right)$ and theoretical results $\left(B_{\mathrm{th}}\right)$ for both models. The two models which include shell effects display a clear reduction in the differences as compared with the Liquid Drop Model LDM1. As decades of work in nuclear masses have taught us, to describe these residual effects is a fairly non-trivial task.

\section{Conclusions}

We have explored the ability of several Liquid Drop Models to describe nuclear binding energies in different deformation regions, and we have performed a similar analysis employing more elaborate models which include shell effects through microscopic terms. We selected for these microscopic corrections the Duflo-Zuker type models and those inspired by the Interacting Boson Model F-spin.

We have shown that the LDM is best suited to describe the masses of prolate deformed nuclei than of spherical ones, while these deformation effects are washed out employing the macroscopic sector of the DZ mass formulas. 

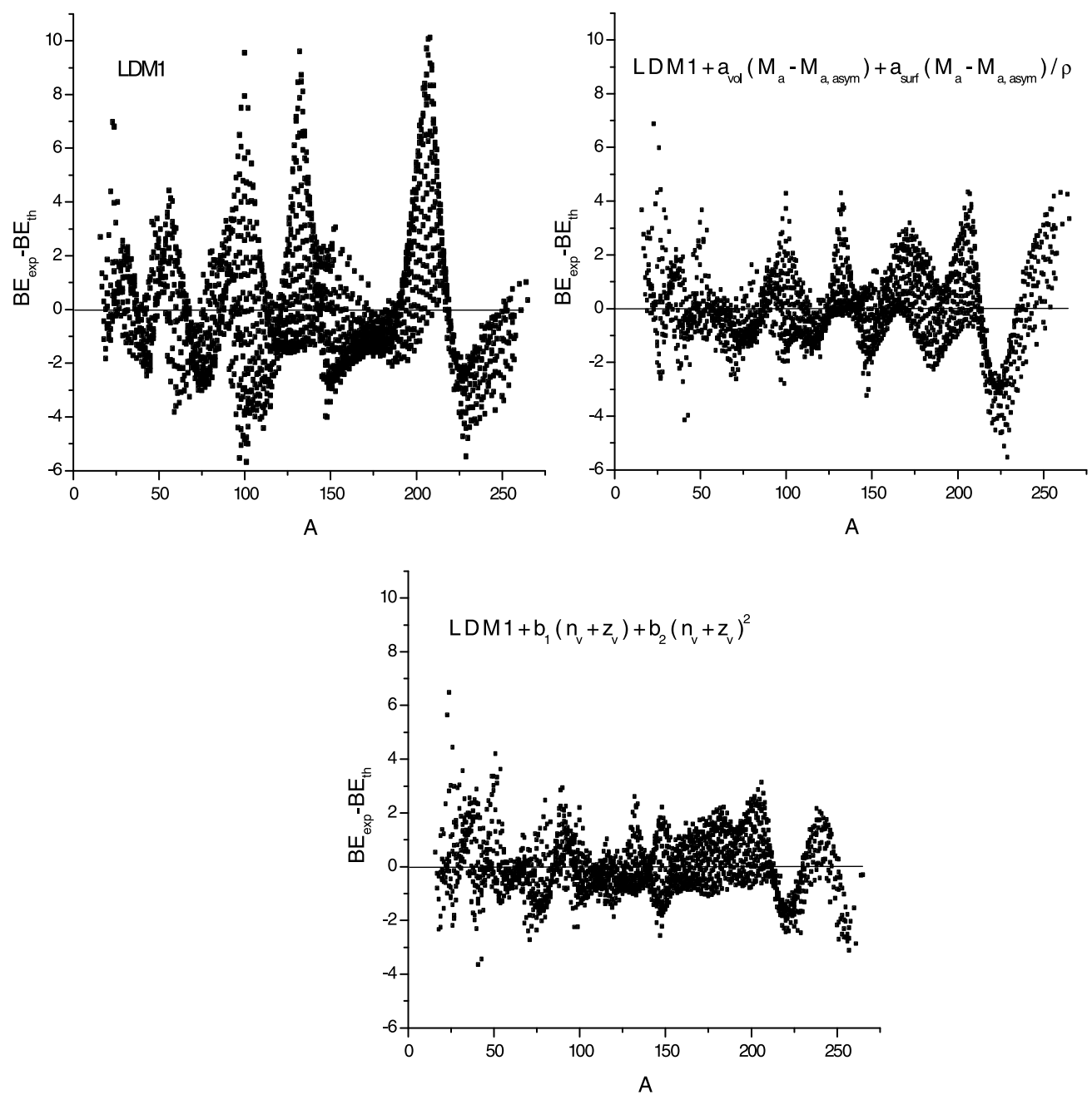

Fig. 8. Comparison between LDM1, LDM1 + DZ and LDM1 + val fits.

We have paid special attention to the DZ master term, which can be used to construct volume and surface terms which describe shell effects. Adding these microscopic information to the LDM formula, we obtain an eight parameter fit of nuclear masses, with a global RMS of $1407 \mathrm{keV}$. The strong dependence of the parameters of the new shell volume and surface terms of the deformation regions could open the possibility to relate shell effects and deformation in a more elaborated way.

\section{Acknowledgements}

C.B. and A.M. are fellows of the CONICET, CCT La Plata (Argentina). J.G.H. thanks A. Aprahamian, O. Civitarese, S. Pittel and P. van Isacker for their valuable comments. This work was supported in part by the Agencia Nacional de Promoción Científica y Tecnológica through 
PICT-2007-00861, Conacyt, México, and DGAPA, UNAM (project 102109), and FONCICyT (project 94142).

\section{Appendix A. Parameters of the fits}

In this appendix we present the parameters found to provide the best fits of the nuclear masses in the nine regions for the mass equations LDM1 (Eq. (1)), LDM2 (Eq. (2)), LDM3 (Eq. (5)), DZ1 (Eq. (7)), DZ2 (Eq. (11)), LDM1 + DZ (Eq. (15)) and LDM1 + val (Eq. (16)).

Table 7

Sets of parameters, in MeV, which minimize the RMS for the nine groups of nuclei, employing LDM1, Eq. (1).

\begin{tabular}{lllllll}
\hline & $a_{v}$ & $a_{s}$ & $a_{c}$ & $a_{p}$ & $S_{v}$ & $y$ \\
\hline set $_{\text {all }}$ & 15.822 & 18.491 & 0.703 & 6.048 & 30.701 & 2.616 \\
set $_{1}$ & 15.515 & 17.577 & 0.686 & 6.092 & 25.997 & 1.583 \\
set $_{2}$ & 15.883 & 18.622 & 0.712 & 5.733 & 28.711 & 2.284 \\
set $_{3}$ & 15.978 & 18.884 & 0.716 & 5.895 & 32.091 & 3.059 \\
set $_{4}$ & 15.879 & 18.564 & 0.708 & 4.103 & 31.459 & 2.666 \\
set $_{5}$ & 15.832 & 18.435 & 0.707 & 5.266 & 30.575 & 2.482 \\
set $_{6}$ & 15.609 & 17.835 & 0.689 & 5.334 & 29.762 & 2.388 \\
set & 15.553 & 17.782 & 0.684 & 5.126 & 27.402 & 1.865 \\
set $_{\text {semi }}$ & 15.775 & 18.012 & 0.712 & 5.500 & 26.407 & 1.489 \\
\hline
\end{tabular}

Table 8

Sets of parameters, in MeV, which minimize the RMS for the nine groups of nuclei, employing LDM2, Eq. (2).

\begin{tabular}{lllllll}
\hline & $a_{v}$ & $a_{S}$ & $a_{c}$ & $a_{\text {pair }}$ & $c_{\text {sym }}$ & $\kappa$ \\
\hline set $_{\text {all }}$ & 15.711 & 18.920 & 0.720 & 6.989 & 30.045 & 1.587 \\
set $_{1}$ & 15.432 & 18.119 & 0.700 & 6.793 & 26.488 & 1.260 \\
set $_{2}$ & 15.695 & 18.802 & 0.722 & 7.443 & 27.979 & 1.441 \\
set $_{3}$ & 15.903 & 19.395 & 0.737 & 6.717 & 30.582 & 1.642 \\
set $_{4}$ & 15.729 & 18.877 & 0.721 & 4.685 & 30.858 & 1.635 \\
set $_{5}$ & 15.685 & 18.756 & 0.721 & 6.210 & 29.969 & 1.548 \\
set & 15.493 & 18.254 & 0.703 & 6.276 & 29.490 & 1.553 \\
set & 15.500 & 18.398 & 0.701 & 6.193 & 27.715 & 1.361 \\
set $_{\text {semi }}$ & 15.627 & 18.329 & 0.724 & 5.327 & 26.043 & 1.099 \\
\hline
\end{tabular}

Table 9

Sets of parameters, in MeV, which minimize the RMS for the nine groups of nuclei, employing LDM3, Eq. (5).

\begin{tabular}{llllllrlrrr}
\hline & $a_{v}$ & \multicolumn{1}{l}{$a_{s}$} & $r_{0}$ & \multicolumn{1}{l}{$a_{\text {pair }}$} & \multicolumn{1}{l}{$k_{v}$} & \multicolumn{1}{c}{$k_{s}$} & \multicolumn{1}{l}{$f_{p}$} & $a_{c, \text { exc }}$ & \multicolumn{1}{c}{$a_{k}$} & \multicolumn{1}{c}{$k_{k}$} \\
\hline set $_{\text {all }}$ & 15.647 & 20.610 & 1.198 & -1.002 & 2.079 & 4.451 & 1.923 & 0.296 & -4.738 & 30.717 \\
set $_{1}$ & 15.834 & 21.074 & 1.203 & -0.905 & 1.305 & -0.812 & 1.265 & 0.304 & -5.910 & -12.666 \\
set $_{2}$ & 15.557 & 20.860 & 1.213 & -1.037 & 1.954 & 4.339 & 2.583 & 0.418 & -4.719 & 32.562 \\
set $_{3}$ & 15.673 & 19.718 & 1.234 & -1.005 & 1.734 & 2.072 & 2.071 & 0.394 & -1.248 & 21.077 \\
set $_{4}$ & 15.671 & 22.448 & 1.227 & -0.726 & 2.720 & 9.059 & 2.936 & 0.661 & -7.069 & 58.552 \\
set $_{5}$ & 15.577 & 21.060 & 1.196 & -0.873 & 2.398 & 6.963 & 2.259 & 0.334 & -6.359 & 44.965 \\
set $_{6}$ & 15.611 & 20.997 & 1.158 & -0.963 & 2.403 & 6.781 & 1.457 & 0.084 & -8.514 & 33.205 \\
set7 $^{15.806}$ & 20.834 & 1.204 & -0.962 & 1.393 & -0.390 & 1.393 & 0.289 & -4.988 & -12.233 \\
set $_{\text {semi }}$ & 15.363 & 18.242 & 1.221 & -0.904 & 1.418 & 0.666 & 2.577 & 0.211 & 0.538 & -0.408 \\
\hline
\end{tabular}


Table 10

Sets of parameters, in MeV, which minimize the RMS for the nine groups of nuclei, employing DZ1, Eq. (7).

\begin{tabular}{|c|c|c|c|c|c|c|}
\hline & $a_{1}$ & $a_{2}$ & $a_{3}$ & $a_{4}$ & $a_{5}$ & $a_{6}$ \\
\hline set $_{\text {all }}$ & 17.531 & 15.433 & 0.694 & 36.157 & 48.425 & 5.154 \\
\hline set $_{1}$ & 17.458 & 15.204 & 0.691 & 33.959 & 39.860 & 5.857 \\
\hline set $_{2}$ & 17.760 & 16.283 & 0.706 & 38.212 & 57.641 & 6.167 \\
\hline set $_{3}$ & 17.788 & 16.329 & 0.708 & 39.263 & 61.183 & 5.807 \\
\hline set $_{4}$ & 17.975 & 16.971 & 0.716 & 39.733 & 61.192 & 6.056 \\
\hline set $_{5}$ & 17.894 & 16.593 & 0.715 & 38.553 & 56.304 & 5.997 \\
\hline $\operatorname{set}_{6}$ & 17.172 & 14.375 & 0.667 & 35.674 & 47.421 & 4.684 \\
\hline set $_{7}$ & 17.379 & 15.037 & 0.682 & 33.507 & 38.541 & 5.206 \\
\hline set $_{\text {semi }}$ & 17.993 & 17.054 & 0.719 & 38.218 & 54.671 & 7.428 \\
\hline
\end{tabular}

Table 11

Sets of parameters, in MeV, which minimize the RMS for the nine groups of nuclei, employing DZ2, Eq. (11).

\begin{tabular}{|c|c|c|c|c|c|c|}
\hline & $a_{1}$ & $a_{2}$ & $a_{3}$ & $a_{4}$ & $a_{5}$ & $a_{6}$ \\
\hline set $_{\text {all }}$ & 15.707 & 16.155 & 0.611 & 29.800 & 34.307 & 7.337 \\
\hline set $_{1}$ & 15.798 & 16.383 & 0.619 & 29.892 & 33.626 & 6.882 \\
\hline set $_{2}$ & 15.801 & 16.357 & 0.620 & 30.997 & 37.886 & 3.960 \\
\hline $\operatorname{set}_{3}$ & 15.535 & 15.684 & 0.599 & 31.938 & 45.543 & 6.855 \\
\hline set $_{4}$ & 16.248 & 17.924 & 0.646 & 31.604 & 36.711 & 8.869 \\
\hline set $_{5}$ & 16.116 & 17.509 & 0.635 & 32.334 & 42.394 & 6.581 \\
\hline $\operatorname{set}_{6}$ & 15.685 & 16.315 & 0.598 & 31.551 & 42.802 & 6.118 \\
\hline set$_{7}$ & 15.381 & 15.076 & 0.589 & 25.663 & 18.329 & 7.087 \\
\hline set $_{\text {semi }}$ & 16.204 & 18.026 & 0.638 & 32.570 & 43.255 & 11.128 \\
\hline
\end{tabular}

Table 12

Sets of parameters, in MeV, which minimize the RMS for the nine groups of nuclei, using LDM1 + DZ, Eq. (15).

\begin{tabular}{|c|c|c|c|c|c|c|c|c|}
\hline & $a_{v}$ & $a_{s}$ & $a_{c}$ & $a_{p}$ & $S_{v}$ & $y$ & $a_{\mathrm{vol}}$ & $a_{\text {surf }}$ \\
\hline set $_{\text {all }}$ & 15.817 & 18.422 & 0.705 & 5.917 & 29.740 & 2.298 & 2.700 & 12.526 \\
\hline set $_{1}$ & 15.727 & 18.142 & 0.700 & 5.893 & 28.721 & 2.090 & 17.792 & -47.797 \\
\hline set $_{2}$ & 15.889 & 18.646 & 0.710 & 4.980 & 32.120 & 2.937 & 18.350 & -48.154 \\
\hline $\operatorname{set}_{3}$ & 15.805 & 18.409 & 0.703 & 5.712 & 33.574 & 3.352 & 20.536 & -52.987 \\
\hline set $_{4}$ & 16.026 & 19.043 & 0.719 & 5.624 & 32.050 & 2.577 & 26.133 & -80.211 \\
\hline set $_{5}$ & 15.911 & 18.717 & 0.711 & 5.731 & 31.079 & 2.532 & 1.962 & 13.575 \\
\hline $\operatorname{set}_{6}$ & 15.703 & 18.103 & 0.696 & 5.297 & 29.991 & 2.368 & -13.570 & 67.354 \\
\hline $\operatorname{set}_{7}$ & 15.515 & 17.641 & 0.682 & 5.267 & 27.509 & 1.894 & -10.468 & 47.078 \\
\hline set $_{\text {semi }}$ & 15.992 & 19.008 & 0.717 & 7.551 & 31.819 & 2.616 & 22.776 & -59.556 \\
\hline
\end{tabular}

Table 13

Sets of parameters, in MeV, which minimize the RMS for the nine groups of nuclei using LDM1 + val, Eq. (16).

\begin{tabular}{lllllllll}
\hline & $a_{v}$ & $a_{S}$ & $a_{c}$ & $a_{p}$ & $S_{v}$ & $y$ & $b_{1}$ & $b_{2}$ \\
\hline set $_{\text {all }}$ & 15.887 & 18.232 & 0.712 & 5.651 & 31.242 & 2.565 & 0.771 & -0.013 \\
set $_{1}$ & 15.805 & 18.067 & 0.707 & 5.882 & 29.052 & 2.073 & 0.606 & -0.008 \\
set $_{2}$ & 15.870 & 18.038 & 0.715 & 4.555 & 29.963 & 2.224 & 0.848 & -0.017 \\
set $_{3}$ & 15.793 & 17.927 & 0.704 & 5.115 & 32.858 & 3.138 & 0.780 & -0.014 \\
set $_{4}$ & 16.075 & 18.789 & 0.725 & 5.086 & 31.544 & 2.401 & 0.730 & -0.012 \\
set $_{5}$ & 15.929 & 18.397 & 0.715 & 5.715 & 31.276 & 2.529 & 0.781 & -0.017 \\
set $_{6}$ & 15.872 & 18.456 & 0.708 & 5.795 & 30.722 & 2.435 & 0.380 & -0.006 \\
set $_{7}$ & 15.678 & 17.850 & 0.695 & 5.083 & 29.023 & 2.146 & 0.456 & -0.009 \\
set $_{\text {Semi }}$ & 16.091 & 18.824 & 0.726 & 6.796 & 31.392 & 2.418 & 0.868 & -0.017 \\
\hline
\end{tabular}




\section{References}

[1] N. Wang, M. Liu, X. Wu, Phys. Rev. C 81 (2010) 044322.

[2] H.A. Bethe, R.F. Bacher, Rev. Mod. Phys. 8 (1936) 82-229.

[3] http://en.wikipedia.org/wiki/Semi-empirical mass formula.

[4] P. Federman, S. Pittel, Phys. Rev. C 20 (1979) 820.

[5] A. Bohr, B.R. Mottelson, Nuclear Structure, vol. I, World Scientific, Singapore, 1998.

[6] C.E. Rolfs, W.S. Rodney, Cauldrons in the Cosmos, University of Chicago Press, Chicago, 1988.

[7] Klaus Blaum, Phys. Rep. 425 (2006) 1.

[8] D. Lunney, J.M. Pearson, C. Thibault, Rev. Mod. Phys. 75 (2003) 1021.

[9] P. Möller, J.R. Nix, W.D. Myers, W.J. Swiatecki, Atom. Data Nucl. Data Tables 59 (1995) 185.

[10] N. Wang, Z. Liang, M. Liu, X. Wu, Phys. Rev. C 82 (2010) 044304.

[11] W.D. Myers, W.J. Swiatecki, Nucl. Phys. A 601 (1996) 141.

[12] K. Pomorski, J. Dudek, Phys. Rev. C 67 (2003) 044316.

[13] S. Goriely, F. Tondeur, J.M. Pearson, Atom. Data Nucl. Data Tables 77 (2001) 311;

S. Goriely, M. Samyn, J.M. Pearson, Phys. Rev. C 75 (2007) 064312.

[14] S. Goriely, N. Chamel, J.M. Pearson, Phys. Rev. Lett. 102 (2009) 152503;

S. Goriely, S. Hilaire, M. Girod, S. Péru, Phys. Rev. Lett. 102 (2009) 242501.

[15] J. Duflo, Nucl. Phys. A 576 (1994) 29.

[16] A.P. Zuker, Nucl. Phys. A 576 (1994) 65.

[17] J. Duflo, A.P. Zuker, Phys. Rev. C 52 (1995) R23.

[18] J. Mendoza-Temis, I. Morales, J. Barea, A. Frank, J.G. Hirsch, J.C. López-Vieyra, P. Van Isacker, V. Velázquez, Nucl. Phys. A 812 (2008) 28.

[19] J. Mendoza-Temis, J.G. Hirsch, A.P. Zuker, Nucl. Phys. A 843 (2010) 14.

[20] J.G. Hirsch, J. Mendoza-Temis, J. Phys. G: Nucl. Part. Phys. 37 (2010) 064029.

[21] A.E.L. Dieperink, P. Van Isacker, Eur. Phys. J. A 32 (2007) 11.

[22] A.E.L. Dieperink, P. Van Isacker, Eur. Phys. J. A 42 (2009) 269.

[23] G. Gangopadhyay, Int. J. Mod. Phys. E 20 (2011) 179.

[24] J.G. Hirsch, C. Barbero, A.E. Mariano, IOP J. Phys.: Conf. Ser. 322 (2011) 012017.

[25] G. Royer, M. Guilbaud, A. Onillon, Nucl. Phys. A 847 (2010) 24.

[26] G. Audi, A.H. Wapstra, C. Thibault, Nucl. Phys. A 729 (2003) 337.

[27] F. James Minuit, Function Minimization and Error Analysis Reference Manual, version 94.1, CERN, 1994, http:// wwwasdoc.web.cern.ch/wwwasdoc/minuit/minmain.html. 